\title{
TRATAMENTO DA SINDROME PARKINSONIANA PELO L-DOPA
}

\author{
Roberto MElaragno * \\ Geraldo M. J. Karam **
}

Em 1960, quando Barbeau ${ }^{1}$ publicou suas observaçōes sôbre o metabolisrıo anormal das catecolaminas nas afecçōes dos gânglios basais, surgiu nova concepção fisiopatogênica para explicar a moléstia de Parkinson. No mesmo ano, Heringer e Hornykiewicz ${ }^{5}$ demonstraram a carência ou a ausência de noradrenalina e dopamina nos núcleos extrapiramidais de pacientes parkinsonianos. Oito anos mais tarde, Cotzias e col. ${ }^{2}$ iniciaram investigações clinico-terapêuticas em parkinsonianos, usando altas doses de levodopa, elemento químico precursor da dopamina, obtendo bons resultados.

Estes fatos tiveram importância pois que o quadro clínico da moléstia de Parkinson até então não tinha sido explicado à luz das alterações anatômicas, seja provocados pela arteriosclerose dos vasos oriundos da artéria cerebral média que irrigam os gânglios basais, seja pelas seqüelas pós-encefalíticas. Na verdade, já em 1953, Greenfield e Bosanquet ${ }^{6}$ haviam demonstrado que a única alteração histopatológica encontrada com freqüência no parkinsonismo era a degeneração da mielina dos neurônios da substância negra, sem entretanto relacioná-la com fatôres de ordem bioquímica. Sabe-se, por outro laāo, que diversas substâncias podem induzir ao estado parkinsoniano ${ }^{4}$, o que vem apoiar, de certo modo, a importância das alterações humorais na sua patogenia.

\section{MATERIAL E MĚTODO}

Estudamos 33 pacientes hospitalizados durante periodos que variaram de uma a oîo semanas. A internação se impôs para melhor avaliação dos dados no que concerne à clasificação clínica, contrôle dos exames laboratoriais, ação terapêtica de dosagens precisas de L-Dopa, evolução dos sinais e sintomas parkinsonianos, estudo dos efeitos colaterais e complicações. O período de internação serviu ainda para aquilatarmos a dose precisa de manutenção com a qual cada paciente deveria seguir na fase ambulatorial.

Os pacientes, operados ou não, eram constituidos por 19 homens e 14 mulheres, com idade variando entre 42 a 78 anos e com diferentes tempos de evoluçāo da moléstia. Os casos foram divididos em 4 grupos segundo a gravidade do quadro clínico, tendo como base uma classificação pessoal para avaliação da sintomatologia, em leves (7 casos), moderados (6), intensos (9) e severos (11).

Serviço de Neurologia do Hospital do Servidor Público de São Paulo: * Chefe do Serviço, Docente da Clínica Neurológica na Universidade de Sāo Paulo; ** Assistente. 
Durante a fase de internação foram feitos o exame clinico geral e neurológico e os seguintes exames complementares: glicemia, dosagens de uréia, ácido úrico, colesterol, reserva alcalina, creatinina, fosfatase alcalina, sódio, potássio, cálcio, proteinas séricas e de bilirrubinas, tempo de atividade de protrombina, atividade de transaminases, hemograma, exame de urina tipo I, eletrencefalograma e eltrocardiograma. Os exames bioquimicos eram repetidos semanalmente em cada paciente; lgumas vêzes foi repetido o eletrocardiograma e solicitada a colaboração de cardioIogista.

O L-Dopa * foi administrado em doses crescentes a partir de $500 \mathrm{mg}$, elevando-se a dose diária de $500 \mathrm{mg}$ cada 3 ou 4 dias, conforme a tolerância de cada paciente, até ser atingido o mínimo de $2 \mathrm{~g}$ e o máximo de $6,5 \mathrm{~g}$ em 24 horas. Em alguns casos, devido a intensas reações tóxicas, foi necessário reduzir a dose e, mesmo, suprimir a medicação, para voltar a administrá-la, após terem sido contornadas tais reações. Apenas em um paciente (caso 25) o tratamento foi abandonado devido a intolerância gástrica, e, principalmente, ao intenso contingente psicogênico.

Com base em razōes farmacodinâmicas, em todos os casos foi utilizado, simultāneamente com o L-Dopa, lim inibidor da MAO (monoaminoxidase) na dose de 3 comprimidos (15 mg) no primeiro dia, sendo a dose diminuida progressivamente até inteira suspensão, o que ocorria em geral até o fim da primeira semana. No que concerne à dieta, apenas fizemos restrição de alimentação hiperprotêica e de compostos vitaminicos do complexo B, sobretudo a piridoxina (vitamina B6), porque êstes produtos reduzem o efeito terapêutico do L-Dopa, por agirem, provàvelmente, acelerando sua descarboxilação nos tecidos extra-cerebrais e diminuindo, assim, seus niveis hemáticos. Todos os pacientes eram submetidos diàriamente à cinesiterapia, mediante exercicios orientados por equipe especializada; à medida que a hiprtonia plástica era diminuída pelo tratamento, a musculatura esquelética, entrando em flacidêz, exigia o emprêgo de exercício ativos para a normalização do tono.

\section{R E S U L T A D O S}

Durante o tratamento, a rigidêz e a marcha dos parkinsonianos se beneficiaram com maior intensidade, comparativamente aos tremores. $\mathrm{Na}$ tabela 1 , em que se avalia a porcentgem global da melhora dos diversos sinais parkinsonianos pelo uso de L-Dopa, verifica-se que a rigidêz melhorou em $38,8 \%$, referidos em valor quantitativo quanto à intensidade do sintoma. Nas tabelas $2,3,4$ e 5 verificamos que a rigidêz melhorou mais nos casos moderados e intensos, respectivamente $43,3 \%$ e $43,8 \%$, em relacão aos casos leves $(35 \%)$ e severos $(33,1 \%)$.

\begin{tabular}{lllllllll}
\hline CASOS & TREMOR & RIGIOEZ & MARCHA & FALA & FACIES & POSTURA & $\begin{array}{c}\text { DEPEMDENCIA } \\
\text { PROPRIA }\end{array}$ & ESCRITA \\
\hline \hline LEVES & 17,5 & 35,0 & 22,5 & 05,0 & 15,0 & $\cdots$ & -1 & 07,0 \\
MODERADOS & 22,5 & 43,3 & 31,6 & 16,0 & 28,3 & 15,0 & 30,0 & 10,0 \\
INTENSOS & 34,1 & 43,8 & 39,4 & 23,5 & 27,7 & 16,1 & 24,3 & 06,6 \\
SEVEROS & 17,2 & 33,1 & 18,7 & 15,9 & 34,0 & 13,7 & 12,7 & 07,3 \\
\hline TOTAL & 22,8 & 38,8 & 28,0 & 15,1 & 26,2 & 14,9 & 22,3 & 07,0 \\
\hline
\end{tabular}

Tabela 1 - Percentagem global quantitativa da melhora dos diversos sinais. A falta de menção de determinados sinais indica a sua ausência.

* Larodopa, fornecido por Produtos Roche Químicos e Farmacêuticos S. A. 


\begin{tabular}{|c|c|c|c|c|c|c|c|c|}
\hline caso & TREMOR & RIGIOEZ & MARCHA & FALA & macies & POSTURA & $\begin{array}{l}\text { DEPENDENCIA } \\
\text { PROPRIA }\end{array}$ & ESCAITA \\
\hline 05 & 30 & 40 & 30 & -- & 20 & -- & -- & 05 \\
\hline 14 & -- & 60 & 60 & 30 & 20 & -- & -- & 10 \\
\hline 23 & 30 & 40 & 20 & 10 & 20 & 10 & 20 & 10 \\
\hline 27 & 10 & 30 & 30 & 20 & 40 & 20 & 40 & 20 \\
\hline 28 & 20 & 40 & 20 & 10 & 40 & -- & - & 05 \\
\hline 36 & - & 50 & 30 & 10 & 30 & $-\infty$ & -- & 10 \\
\hline TOTAL & 22,5 & 43,3 & 31,6 & 16 & 28,3 & 15 & 30 & 10 \\
\hline
\end{tabular}

Tabela 2 - Percentagem de melhora dos diversos sinais nos casos considerados como moderados.

\begin{tabular}{lllllllll}
\hline CASO & TREMOR & MIGIDEZ & MARCHA & FALA & FAGIES & POSTURA & $\begin{array}{c}\text { DEFEROENCIA } \\
\text { PMPRAA }\end{array}$ & ESCRTA \\
\hline 12 & 50 & 50 & 40 & 30 & 30 & 10 & 20 & 05 \\
13 & -- & 60 & 50 & 60 & 40 & 20 & 40 & 10 \\
18 & 40 & 80 & 80 & 20 & 60 & 50 & 60 & 10 \\
24 & 05 & 20 & 20 & -- & 10 & 10 & 10 & 05 \\
25 & -- & 05 & 05 & 05 & 10 & 05 & 05 & 05 \\
29 & 30 & 40 & 30 & - & 20 & 10 & -- & 10 \\
30 & 30 & 40 & 40 & 30 & 30 & 10 & 10 & 05 \\
31 & - & 40 & 50 & 10 & 30 & 10 & 20 & 05 \\
35 & 50 & 60 & 40 & 10 & 20 & 30 & 30 & 05 \\
\hline TOTAL & 34,1 & 43,8 & 39,4 & 23,5 & 27,7 & 16,1 & 24,3 & 06,6 \\
\hline
\end{tabular}

Tabela 3 - Percentagem de melhora dos diversos sinais nos casos considerados como intensos.

A marcha melhorou em média $28 \%$ (tabela 1), coerentemente com a melhora da rigidez; entretanto, nos vários gráus de intensidede, a melhora da marcha foi mais acentuada nos casos moderados (31,6\%) e intensos $(39,4 \%)$, restando $22,5 \%$ para os casos leves e $18,7 \%$ para os severos. Estes dois últimos resultados mais baixos são compreensiveis em vista da nossa classificacão atribuir, aos casos leves, marcha normal ou com discreta perda dos movimentos associados e, aos casos severos, marcha impossivel ou com intensa dificuldade, sempre com o auxilio de terceiros. A rigidez facial ou hipominia melhorou ràpidamente e constituiu o mais precoce dos efeitos terapéuticos da L-Dopa. 


\begin{tabular}{|c|c|c|c|c|c|c|c|c|}
\hline CAsO & TREMOR & Rieiocz & MARCHA & IALA & racies & mostuna & $\begin{array}{l}\text { DEPEMDEMEIA } \\
\text { PROPAIA }\end{array}$ & Escemin \\
\hline 1 & 20 & 40 & 20 & -- & 10 & -- & - & 05 \\
\hline 2 & - & 20 & 20 & 05 & 20 & - & -- & - \\
\hline 4 & 10 & - & - & -- & -- & -- & -- & - \\
\hline 11 & -- & -- & -- & - & - & -- & -- & 10 \\
\hline 22 & 10 & -- & -- & -- & - & - & -- & 05 \\
\hline 26 & 30 & 40 & 20 & -- & 10 & -- & -- & 10 \\
\hline 33 & -- & 40 & 30 & -- & 20 & -- & -- & 05 \\
\hline TOTAL & 17,5 & 35 & 22,5 & 05 & 15 & -- & -- & 07 \\
\hline
\end{tabular}

Tabela 4 - Percentagem de melhora dos diversos sinais nos casos considerados como leves.

\begin{tabular}{|c|c|c|c|c|c|c|c|c|}
\hline caso & Themon & migiocz & MARCHA & FALA & racies & PCstuna & $\begin{array}{c}\text { DEPENDENCIA } \\
\text { PROPRIA }\end{array}$ & ESCRITA \\
\hline 3 & 20 & 40 & 30 & 10 & 20 & 10 & 05 & -- \\
\hline 6 & 05 & 70 & 80 & 70 & 60 & 30 & 50 & 20 \\
\hline 7 & 02 & 20 & 01 & 05 & 60 & 01 & - & -- \\
\hline 8 & 30 & 50 & 30 & 10 & 60 & 20 & 20 & 05 \\
\hline 10 & 20 & 40 & 15 & 20 & 40 & 20 & 10 & 20 \\
\hline 19 & 10 & 10 & 10 & 10 & 40 & 05 & 10 & 05 \\
\hline 20 & 05 & 15 & 10 & 10 & 30 & 10 & 10 & 05 \\
\hline 21 & -- & 20 & 10 & 10 & 10 & 05 & 05 & 01 \\
\hline 32 & 40 & 50 & 10 & 10 & 20 & 10 & 10 & 05 \\
\hline 34 & -- & -- & -- & -- & 05 & -- & -- & -- \\
\hline 40 & 40 & 50 & 10 & 20 & 30 & 40 & 20 & 20 \\
\hline TrTaL & 17.2 & 33,1 & 18.7 & 159 & 34 & 13.7 & 12.7 & 07,3 \\
\hline
\end{tabular}

Tabela 5 - Percentagem de melhora dos diversos sinais nos casos considerados como severos.

Os tremores figuram a seguir (Tabela 1), com a percentagem de $22,8 \%$ e tiveram maiores beneficios nos casos intensos $(34,1 \%)$, relativamente aos casos leves $(17,5 \%)$. Os casos severos tiveram melhora de $17,2 \%$ e, os moderados, de $22,5 \%$. Quanto ao estado de dependência própria, ou seja, os cuidados de autonomia dos 
pacientes para a execuçāo dos movimentos úteis ao asseio corporal e a se alimentar, verificamos melhora global de 22,3\%. Para os casos leves, não há referências, pois que êstes pacientes, por definição, têm perfeita autonomia de movimentos para a execução dos cuidados pessoais. Em relação ao gráu de dependência própria, os pacientes melhoraram tanto mais acentuadamente quanto mais benignos eram seus quadros clínicos: $30 \%, 24,3 \%$ e $12,7 \%$ respectivamente para os casos moderados, intensos e severos.

Com respeito à fala, foi verificada uma melhora global de $15,1 \%$ nos 4 grupos, sendo que os pacientes com sintomatologia intensa obtiveram melhora mais evidente, $(23,5 \%)$, em relação aos de grupo leve $(5 \%)$, moderado (16\%) e severo $(15,9 \%)$. Estes resultados aparentemente dispares não carecem de lógica pois que os pacientes do grupo leve e moderado, pela nossa classificação, não têm muito que melhorar e, nos de grupo severo, a fala é quase impossivel ou ininteligivel. Além disso, assinalamos menores resultados de me!horia da fala em pacientes disártricos nos quais fôra feita talamotomia.

A postura de nossos pacientes não respondeu na mesma propoìção que os demais sinais e sintomas clinico neurológicos: a porcentagem global de melhora foi pequena $(14,9 \%)$ e a diferença entre os vários grupos foi minima. Quanto à escrita, a melhora global foi inexpressiva $(7,0 \%)$ sendo que nos casos moderados atingiu $10 \%$.

kepercussōes laboratoriais - $\mathrm{Na}$ análise dos resultados labora+oriais, lançamos os resultados em gráficos, num sistema de coordenadas cartesianas onde, nas abcissas figuram o tempo e a dose de L-Dopa e, nas ordenadas, os resultados dos exames em miligramos/por cento. Assim notamos que a uréia, dosada pelo método de auto-analyser, e o ácido úrico, dosado pelo método de Caraway, apresentaram tendência para as elevaçōes, sobretudo naqueles casos que apresentaram grandes reações tóxicas. A reserva alcalina, dosada pelo método de Oxford, evidenciou discreta tendência para queda. As demais dosagens bioquímicas não mostraram alteraçōes. No que concerne ao hemograma, ocorreu, por vêzes, depressão da série eritrocitária em seu número global, assim como ligeira hipocromia, que foram corridas com medicamentos à base de suífato ferroso; em nenhum dêstes casos, foram necessárias transfusōes sangüineas. A série leucocitária só se alterou na vigência de processo infeccioso intercorrente, com neutrofilia e desvio para a esquerda (casos 4 e 7), concluindo-se que o L-Dopa não inibe êste setor reacional da medula. Com referência aos elementos figurados do sangue, foram assinalados por vêzes intensas eosinofilias.

Efeitos colaterais - No que diz respeito aos efeitos colaterais durante o tratamento pelo L-Dopa, distinguimos dois tipos clinicamente bem distintos:

A) Ação adrenérgica, surgindo na primeira semana de tratamento, na vigência da administração do inibidor da MAO concomitantemente com o L-Dopa (Tabela 6). Nesta fase observamos intensa ação simpaticotônica da adrenalina e da noradrenalina, manifestando-se clinicamente por cefaléia, hipertensão arterial, rubor facial, sudorese, tenesmo vesical e angina pectoris. A ação adrenérgica foi, por vêzes, tão intensa que, em três casos $(6,7$ e 24), as dosagens de ácido vanil-mandélico (método de Giltlow modificado sob regime hipofenólico) ocorreu resposta do tipo feocromocitoma. Este fato é explicado pela ação inibitória da MAO que bloqueia, juntamente com o OMT (O. metil transferase), a via de degradação da dopamina em seus metabolitos principais, o ácido homovanilico e a trimetoxitiramina. Desta forma, além de preservar o estoque de dopamina nos núcleos da base, facilita-se a via de sintese das outras catecolaminas (adrenalina e noradrenalina) as quais, caindo na circulação em grande quantidde, resultam no efeito adrenérgico intenso. No cômputo global dos quatro grupos, o rubor facial predominou com $70,45 \%$ enquanto que, nas avaliações parciais, obtivemos maior porcentagem para os casos leves $(85 \%)$. A cefaléia foi também bastante evidente (65\% no cômputo geral dos casos), sendo que, nos casos leves, ocorreu em $100 \%$ dos casos. A sudorese figurou 


\begin{tabular}{|c|c|c|c|c|c|c|c|}
\hline & CASO & $\begin{array}{c}\text { HIPEATENSÄO } \\
\text { ARTERIAL }\end{array}$ & CEFALEIA & RUBOR S & SUDORESE & $\begin{array}{l}\text { TENESANO } \\
\text { VESICAL }\end{array}$ & $\begin{array}{l}\text { ANGINA } \\
\text { PECTORIS }\end{array}$ \\
\hline \multirow[t]{7}{*}{ LEVES } & 1 & $++t$ & $++t$ & $+t$ & + & $\overline{-}$ & $+t+t$ \\
\hline & 2 & ++ & $+t$ & $+t$ & - & $+t$ & - \\
\hline & 4 & + & ++++ & ++ & + & - & - \\
\hline & 11 & + & ++ & - & - & - & - \\
\hline & 22 & + & +++ & ++ & - & - & - \\
\hline & 26 & - & + & + & - & - & - \\
\hline & & - & + & $+t$ & - & - & - \\
\hline \multirow{8}{*}{ MODERADO } & & $71 \%$ & $100 \%$ & $85 \%$ & $42,7 \%$ & $14,2^{\circ}$ & $\% 14,2 \%$ \\
\hline & 5 & + & ++ & ++ & ++ & - & - \\
\hline & 14 & - & - & + & - & - & - \\
\hline & 23 & - & - & - & - & - & - \\
\hline & 27 & - & + & + & - & - & - \\
\hline & 28 & - & + & ++ & ++ & - & - \\
\hline & 36 & - & - & - & + & - & - \\
\hline & & $16,6 \%$ & $50 \%$ & $66,6 \%$ & $\% 50 \%$ & $0 \%$ & $0 \%$ \\
\hline \multirow[t]{10}{*}{ INTENSOS } & 12 & - & - & + & - & - & - \\
\hline & 13 & - & + & - & - & - & - \\
\hline & 18 & + & $+t+$ & $++t$ & + & - & - \\
\hline & 24 & - & - & + & - & - & - \\
\hline & 25 & + & ++ & ++ & + & +++ & +- \\
\hline & 29 & - & - & + & $+t$ & - & - \\
\hline & 30 & - & + & - & - & - & - \\
\hline & 31 & + & - & - & - & - & - \\
\hline & 35 & + & $+t$ & $+t$ & + & - & - \\
\hline & & $36 \%$ & $55,5 \%$ & $66,6 \%$ & $\% 44,4 \%$ & $11,1 \%$ & $60 \%$ \\
\hline \multirow[t]{11}{*}{ sEvenos } & 3 & - & ++ & + & + & - & - \\
\hline & 6 & + & +++ & $+t$ & ++ & + & - \\
\hline & 7 & +++ & +++ & +++ & +++ & $+t$ & - \\
\hline & 8 & ++ & - & $+t$ & + & - & - \\
\hline & 10 & - & - & - & + & - & - \\
\hline & 19 & ++ & ++ & ++ & ++ & + & - \\
\hline & 20 & - & + & - & - & - & - \\
\hline & 21 & - & - & - & - & - & - \\
\hline & 32 & - & + & + & + & - & - \\
\hline & 34 & $\bar{t}$ & - & + & ++ & $i_{t+1}$ & $=$ \\
\hline & 40 & + & - & - & + & +++ & - \\
\hline
\end{tabular}

Tabela 6 - Ação adrenérgica observada na primeira semana de tratamento pelo uso de inibidor MAO.

em 54,72\% no cômputo global dos casos; entretanto, causou espécie o fato dêste sintoma se apresentar de modo mais expressivo nos casos severos $(81,8 \%)$ em relaçāo aos demais casos. A hipertensão arterial figurou com $42,2 \%$ no cômputo global e, na tabela diferencial dos grupos, predominou nos casos leve com $71 \%$. Em menor porcentagem figuraram o tenesmo vesical e a angina pectoris, na consideraçāo global dos quatro grupos $(13,1 \%$ e $3,55 \%$ respectivamente). O caso 1 apresentou um quadro de angina pectoris bem caracterizado; entretanto, vencida a crise, foi reiniciada a administraçāo do L-Dopa, sem o uso de inibidor da MAO Em resumo, as reações adrenérgicas predominaram nos casos leves, o que faz supor que, nestes pacientes, o metabolismo da fenilalanina estaria ainda relativamente preservado, respondendo com ma1or intensidade à ação farmacodinâmica dos medicamentos. As reações adrenérgicas surgiam 30 minutos a 2 horas após a tomada do L-Dopa, quando associado ao inibidor da MAO. Neste periodo, para atenuar os sintomas desagradáveis, mantinhamos os pacientes em decúbito elevado, com medicação analgésica, sem empregar hipotensores.

B) Ação dopaminérgica - Esta ação colateral se evidencia após a suspensão do inibidor da MAO, por conseguinte 10 a 12 dias depois do inicio do tratamento. Provàvelmente, esta fase principia logo no inicio do tratamento pelo L-Dopa. Entretanto, 
nesse primeiro periodo, as reaçōes adrenérgicas seriam tão intensas a ponto de mascarar as reaçōes dopaminérgicas. As reaçōes dopaminérgicas se caracterizam por hipertensāo arterial, sudorese fria, arritmia cardiaca e lipotímia. No cômputo global dos 4 grupos (Tabela 7) figura a hipotensão arterial como sinal de maior intensidade $(45,7 \%)$, seguido da lipotimia $(41,69 \%)$, sudorese fria $(29,15 \%)$ e, finalmente, arritmia cardíca $(\mathbf{1 7 , 5 \% )}$. Na classificaçāo parcelada dos 4 grupos verificamos que a hipotensāo arterial prevaleceu, quanto à intensidade, nos casos severos $(90,9 \%)$. A sudorese fria e a lipotimia, que possivelmente decorrem diretamente da hipotensão arterial, figuram ambas com $63,3 \%$ para os casos severos. Em resumo, observamos que a açāo dopaminérgica foi mais intensa nos casos de maior gravidade, em relaçāo aos mais benígnos. Este comportamento pode ser explicado pelo fato de os pacientes mais intensamente afetados haverem recebido doses mais elevadas de L-Dopa em relaçāo aos demais.

\begin{tabular}{|c|c|c|c|c|c|c|}
\hline & CASO & $\begin{array}{l}\text { DOSACEM DE } \\
L \text { DOPA }\end{array}$ & $\begin{array}{l}\text { HIPOTENSIO } \\
\text { ARTERIAL }\end{array}$ & $\begin{array}{c}\text { SUDORESE } \\
\text { FRIA }\end{array}$ & AARITMIA & LIPOTIMIA \\
\hline LEVES & $\begin{array}{r}1 \\
2 \\
4 \\
11 \\
22 \\
26 \\
33 \\
\end{array}$ & $\begin{array}{l}2,0 \mathrm{gr} . \\
2,5 \mathrm{gr} . \\
4,0 \mathrm{gr} . \\
3,0 \mathrm{gr} . \\
4,5 \mathrm{gr} . \\
2,5 \mathrm{gr} . \\
3,0 \mathrm{gr} .\end{array}$ & $\begin{array}{l}- \\
\pm \\
\overline{-} \\
\overline{-}\end{array}$ & $\begin{array}{l}- \\
\pm \\
\pm \\
-\end{array}$ & $\begin{array}{l}+ \\
- \\
- \\
- \\
- \\
-\end{array}$ & $\begin{array}{l}- \\
\pm \\
- \\
- \\
-\end{array}$ \\
\hline MODERADOS & $\begin{array}{r}5 \\
14 \\
22 \\
27 \\
28 \\
36\end{array}$ & $\begin{array}{l}40 \mathrm{gr} . \\
3,5 \mathrm{gr} . \\
3,0 \mathrm{gr} . \\
3,0 \mathrm{gr} . \\
5,5 \mathrm{gr} . \\
2,5 \mathrm{gr}\end{array}$ & $\begin{array}{c}14,2 \% \\
+ \\
\pm \\
\pm \\
- \\
-\end{array}$ & $\begin{array}{c}14,2 \% \\
= \\
+ \\
= \\
-\end{array}$ & $\begin{array}{c}14,2 \% \\
\pm \\
= \\
- \\
-\end{array}$ & $\begin{array}{c}14,2 \% \\
\pm \\
+ \\
- \\
-\end{array}$ \\
\hline IHTENSOS & $\begin{array}{l}12 \\
13 \\
18 \\
24 \\
25 \\
29 \\
30 \\
31 \\
35\end{array}$ & $\begin{array}{c}40 \mathrm{gr} . \\
2.0 \mathrm{gr} . \\
3,0 \mathrm{gr} \\
40 \mathrm{gr} \\
\text { abandonow } \\
3.0 \mathrm{gr} \text {. } \\
4,5 \mathrm{gr} \text {. } \\
4,0 \mathrm{gr} \text {. } \\
4,0 \mathrm{gr} .\end{array}$ & $\begin{array}{c}33,3 \% \\
+ \\
+ \\
+ \\
= \\
= \\
- \\
+\end{array}$ & $\begin{array}{c}16,6 \% \\
+ \\
+ \\
+ \\
= \\
= \\
- \\
-\end{array}$ & $\begin{array}{c}16.6 \% \\
= \\
\pm \\
= \\
= \\
- \\
-\end{array}$ & $\begin{array}{c}33,3 \% \\
+ \\
+ \\
+ \\
+ \\
= \\
- \\
+\end{array}$ \\
\hline SEVEROS & $\begin{array}{r}3 \\
6 \\
7 \\
8 \\
10 \\
19 \\
20 \\
21 \\
32 \\
34 \\
40\end{array}$ & $\begin{array}{r}6,0 \mathrm{gr} . \\
4,0 \mathrm{gr} . \\
4,0 \mathrm{gr} . \\
4,0 \mathrm{gr} \\
1,5 \mathrm{gr} . \\
4,0 \mathrm{gr} . \\
3,5 \mathrm{gr} . \\
3,0 \mathrm{gr} . \\
4,0 \mathrm{gr} \\
\text { abandow } \\
1,0 \mathrm{gr} .\end{array}$ & $\begin{array}{c}44,4 \% \\
+ \\
+ \\
+ \\
+ \\
+ \\
+ \\
+ \\
+ \\
+ \\
+ \\
+\end{array}$ & $\begin{array}{c}22,2 \% \\
+ \\
+ \\
+ \\
+ \\
= \\
+ \\
= \\
+ \\
+\end{array}$ & $\begin{array}{c}11,1 \% \\
+ \\
- \\
- \\
+ \\
- \\
= \\
= \\
+\end{array}$ & $\begin{array}{c}55,5 \% \\
+ \\
+ \\
+ \\
+ \\
+ \\
- \\
- \\
+ \\
+ \\
+\end{array}$ \\
\hline
\end{tabular}

Tabela $y$ - Ação dopaminérgica observada após quatro semanas de tratamento pelo L-Dopa.

Complicaçōes - As complicaçōes clinicas gerais observadas durante o tratamento foram: anorexia, obstipação intestinal, náuseas, vómitos, depressāo psiquica, edema e euforia. A anorexia prevaleceu com a média de 75,5\% determinando emagrecimento dos pacientes o que, de certo modo, facilitava as condições cinesiterápicas e de locomoçāo durante a marcha. A obstipacão intestinal figurou com $68,8 \%$ posslvelmente agravando, durante o tratamento pelo L-Dopa, um sintoma freqüente e próprio da moléstia. Náuseas e vômitos ocorreram em 56,2\% e 46,8\% dos casos, 
respectivamente. Estes sintomas eram, em geral, contornados por anti-eméticos ou pela administração do L-Dopa após as refeições. Os estados psiquicos de depressão e de euforia foram assinalados em $46 \%$ e $6,4 \%$ dos casos, respectivamente. Os ede.mas dos membros inferiores foram discretos, ocorrendo em $11,8 \%$ dos pacientes, comprometendo preponderantemente os pacientes mais idosos.

Complicacões neurológicas - As manifestações de super-dosagem de L-Dopa se expressam cllnicamente como manifestações extrapiramidaís iatrogênicas. Além disso, observou-se também um estado de prostração que se manifestava por debilidade geral ou, por vêzes, quase estado comatoso, eventualmente com mialgias. Nestas eventualidades, suspendiamos a medicação e instituiamos a hidratação com soro glicosado, associado a $2 \mathrm{~g}$ de vitamina $\mathrm{C}$ e à corticoterapia. Depois do desaparecimento dos sintomas reiniciavamos o tratamento pelo L-Dopa, em doses mals lentamente progressivas. Após 24 a 48 horas o estado dito parkinsonóide, que se caracterizava pela volta ou exacerbação dos tremores ou da rigidez, atingiu 27,3\% dos pacientes tratados. Quedas foram também observadas e talvez fossem consequiência da acinesla e da hipotensão arterial postural, atingindo 23,2\%. As discinesias, em porcentagem de $20,6 \%$, surgiram como movimentos involuntários da língua e da mandibula, torcicolos ou espasmos faciais. Este tipo de reação obrigava também à suspensâo ou diminuicão da droga. Em todos os casos de acinesia ou discinesia, após a interrupçāo do tratamento, o seu reinicio se processava sem as manifestacões tóxicas anteriormente ocorridas, mesmo quando se ultrapassava aquela dose que havia determinado o quadro tóxico iatrogênico. Finalmente a acatisia foi a manifestação mais rara, sendo assinalada em 12,2\% dos pacientes. Este estado também obrigou à reduçāo da dose de L-Dopa para, após 24 ou 48 horas reiniciar a medicaçāo com aumento progressivo.

\section{R E S U M O}

O trabalho refere o tratamento com L-Dopa de 33 pacientes internados, sendo 19 homens e 14 mulheres. Os casos foram agrupados, segundo uma classificação esquemática, em leves, moderados, intensos e severos. As idades dos pacientes, previamente operados ou não, variaram de 42 a 78 anos. $O$ esquema de medicação constou de administração de L-Dopa na dose inicial de $500 \mathrm{mg}$ associado a um inibidor da MAO. A dose de L-Dopa era elevada de $500 \mathrm{mg}$ cada 3 ou 4 dias, segundo a tolerância de cada paciente. Todos os sintomas dependentes, direta ou indiretamente, da rigidez tiveram melhoras mais nítidas em relação aos tremores. Durante a administração do medicamento os teores sangüíneos de ácido úrico e uréia, mostraram tendência para se elevar; a reserva alcalina, pelo contrário, tendeu para a diminuição; o hemograma revelou muitas vêzes eosinofilia por vêzes intensa. Os efeitos colaterais foram catalogados em dois tipos - efeitos adrenérgicos e efeitos dopaminérgicos - correspondentes a duas fases: uma primeira, de pequena duração, na qual foram usados concomitantemente o L-Dopa e um inibidor da MAO, surgindo cefaléia hipertensão arterial paroxística, rubor facial, sudorese, tenesmo vesical e angina pectoris; numa segunda fase surgiram os efeitos colaterais ditos dopaminérgicos, proporcionais à gravidade clínica do caso, cujas manifestações principais foram hipotensão arterial, sudorese fria, arritmia cardíaca e lipotímia. As complicações clínicas gerais eram representados habitualmente por anorexia, obstipação intestinal, náuseas, vômitos, discretas alterações do estado psíquico com depressão e euforia. 
Dentre as complicações neurológicas foram assinalados o estado parkinsonóide, discinesias e acatisia.

S U M M A R Y

\section{Parkinsonism's treatment by L-Dopa}

The results of the treatment with L-Dopa of 33 patients (19 males and 14 females) are reported. The cases were separated, acording to their symptoms and signs, in slight, moderate, intense and severe ones. Age of the patients : between 42 and 78 years old. L-Dopa was administered in an initial dosis of $500 \mathrm{mg}$, associated to a MAO inhibitor; the dosis was increased $500 \mathrm{mg}$ every 3 or 4 days, according to the patient's tolerance. There was a better improvement on all the phenomena dependent directly or indirectly of the rigidity, as related to the tremor. During the treatment the blood values of uric acid and urea had a tendency to increase; alkalin reserve had a tendency for lowering; the leucocyte count revealed an eosiniphilia which was sometimes very high. Collateral effects (adrenergic and dopaminergic) were analysed. The adernergic effects occured when L-Dopa was used associated to a MAO inhibitor and were represented by headache, paroxysmal arterial hypertension, facial rubor, profuse sweating, vesical tenesmus and angina pectoris. The dopaminergic effects, proportional to the clinical gravity of the case, were: arterial hypotension, coldsweating, cardiac arrytmia and lipothimia. Other complications were: anorexia, decrease of bowel movements, nausea, vomiting, psychic alterations with depression and euphoria. Among the neurologic complications are emphasized discinesias and acatisia.

\section{REFER E N I A S}

1. BARBEAU, A. - Preliminary observation on abnormal catecholamine metabolism in basal ganglia diseases. Neurology (Minneapolis) 10:446, 1960.

2. COTZIAS, G. C.; PAPAVASIluOU, P. S. \& GELlENE, R. - Experimental treatment of parkinsonism with L-Dopa. Neurology (Minneapolis) 18:276, 1968.

3. DEGKWITZ, R.; FROWEIN, R.; KULENKAMPFF, C. \& MOHS, U. - Ueber die Wirkungen des L-Dopa beim Menschen und deren Beeiflussung durch Reserpin Chlorpromazin, Iproniazid und vitamin $B_{6}$. Klin. Wschr. 38:120, 1960.

4. DUVOISIN, R.; YAHR, M. D.; SCHEAR, M.; HOEHN, M. \& BARRET, R. E. The present status of L-Dopa in the treatment of parkinsonism. Arch. Neurol. (Chicago) 21:343, 1969.

5. HERINGER, H. \& HORNYKIEWICZ, O. - Verteilung von Noradrenalin und Dopamin (3- Hydroxytyramin) in Gerhin des Menschen und ihren verhalten bei Erkrankungen des extrapyramidalen Systems. Klin. Wschr. 38:1236, 1960.

6. GREENFIELD, J. G. \& BOSANQUET, F. D. - The brainstem lesions in parkinsonism. J. Neurol. Neurosurg. Psychiat. 16:213, 1953.

Hospital do Servidor Público Estadual - Rua Pedro de Toledo 1800 - São Paulo, SP - Brasil. 